\title{
Review on the application of freeway CVIS communication technology
}

\author{
Shouyan $\mathrm{Fu}^{1}$, Wenhong $\mathrm{Lv}^{1, \mathrm{a}}$, Jiali $\mathrm{Ge}^{2}$ and Yanxi $\mathrm{Qu}^{3}$ \\ ${ }^{1}$ College of Transportation, Shandong University of Science and Technology, China \\ 2 Shandong Cultural Industry Vocational College, China; \\ ${ }^{3}$ College of Electronic Information Engineering, Shandong University of Science and Technology, China
}

\begin{abstract}
This paper mainly describes the research progress of Cooperative Vehicle Infrastructure System (CVIS) communication technology in freeway. The application status of short-range real-time communication technology and intermediate-range interactive communication technology in different scenarios of freeway CVIS are discussed respectively. This paper summarizes the existing problems in the research of CVIS communication technology from two aspects of technology application and network security. The research shows that in the future, machine learning, deep learning and other methods can be used to automatically detect network intrusion and deal with unknown attacks; In the future, the CVIS communication technology will adopt channel coding technology and encryption technology to improve the security of wireless network; With the development of 5G technology, the Internet of Vehicles(IOV) will usher in new opportunities. In the future, the freeway communication network will be a heterogeneous wireless network with multiple access networks, which can meet the application requirements in different road traffic scenarios, effectively promote the construction of freeway informatization and accelerate the construction process of smart freeway.
\end{abstract}

\section{Introduction}

Vehicle-mounted communication and network is one of the key technologies of Cooperative Vehicle Infrastructure System (CVIS). CVIS communication mainly includes Vehicle-to-Vehicle (V2V), Vehicle-toInfrastructure (V2I) and Vehicle-to-Pedestrian (V2P). In the CVIS, the communication technology should ensure the high reliability communication between V2V and V2I, and ensure the timely sending and receiving of vehicle safety information, early warning information and road status information. However, considering the communication performance, network security, application scenario and cost, it is difficult for a single communication technology to fully meet the above requirements. This paper summarizes the domestic and foreign literature, describes the characteristics of short-range real-time communication technology and intermediate-range interactive communication technology and their application status in different scenarios of CVIS; points out the problems existing in the research of CVIS communication technology at the present stage; and analyses the development trend of CVIS communi- cation technology, aiming to provide reference for the future construction of CVIS with multi-network access and fusion.

a Corresponding author: zklwh@sdust.edu.cn

\section{Application research status of freeway CVIS communication technology}

According to different communication objects and communication distances, the freeway CVIS communication technology is divided into short-range real-time communication and intermediate-range interactive communication [1]. The main application scenarios are shown in Table 1.

\subsection{Application research of short-range real-time communication technology}

Short range real-time communication, namely shortdistance emergency communication, is suitable for $\mathrm{V} 2 \mathrm{~V}$ and V2I communication. It mainly transmits collision warning, emergency road condition, emergency call for help and other data and information related to traffic safety [2]. The amount of such information data is not large, but the real-time communication requirements are high.

Bluetooth is suitable for occasions where the communication rate is not high. The application of Internet of Vehicles mainly includes two aspects [3]. One is to use Bluetooth as a component of vehicle embedded terminal to provide information service; the other is to use Bluetooth device as vehicle identification to detect 
vehicle status information through roadside equipment [4, 5]. RFID can be divided into four categories according to the working frequency. The RFID tags in ultrahigh frequency $(860 \mathrm{M} \sim 960 \mathrm{MHz})$ and microwave $(2.4 \mathrm{GHz}$, $5.8 \mathrm{GHz}$ ) bands are mainly used in the traffic field. ZigBee technology has the characteristics of point -to-point two-way communication and wireless ad-hoc network, and it is mainly applied to vehicle collision prevention system and road environment monitoring in vehicle networking. Although many scholars have made some achievements in the research of V2X security application based on DSRC, the channel occupancy rate will be greatly increased in the case of highly dense vehicles, which will lead to the performance degradation of IEEE802.11. Moreover, in DSRC spectrum, the bandwidth allocated by $\mathrm{V} 2 \mathrm{~V}$ security application is limited, which is not enough to meet the demand of $\mathrm{V} 2 \mathrm{~V}$ excessive service in the future. In V2I communication, DSRC is mainly used for non-secure applications, such as freeway toll collection [6], download of electronic map and traffic scheduling, etc. At present, DSRC has been relatively mature in the application of ETC. The characteristics and applicable scenarios of short-range communication technology above are shown in Table 2.

\subsection{Application research of medium-range interactive communication technology}

The intermediate-range interactive communication technology is mainly applied to the communication between vehicles and infrastructure. It mainly transmits real-time information such as vehicle congestion, dynamic speed limit, temporary danger prompt, video and video download, which can improve the traffic efficiency and driving comfort, but it requires high transmission bandwidth. WiFi is a series of network protocols based on IEEE 802.11. In shorter distance V2V and V2I communication, WiFi has higher throughput and lower latency than WiMAX [7]. The development of cellular mobile communication network has experienced $1 \mathrm{G}, 2 \mathrm{G}$, $3 \mathrm{G}, 4 \mathrm{G}$ until now $5 \mathrm{G}$ communication. LTE and $5 \mathrm{G}$ are the most promising technologies in CVIS. Compared with WAVE, LTE has the advantages of high network capacity and wide coverage, but it has higher delay when the network load increases. It is suitable for high bandwidth demand and QoS sensitive vehicle applications, namely information entertainment. Compared with the the previous cellular networks, 5G technology has characteristics of low delay, high capacity, super height and high reliability [8]. ITU divides 5G network service into three application scenarios, among which high reliability and low delay communication (URLLC) is mainly used in Internet of Vehicles. At present, the application of $5 \mathrm{G}$ in the Internet of Vehicles is still in the testing stage and has not been put into use formally. The characteristics and specific application scenarios of the above intermediate-range communi- cation technologies are shown in Table 3.

Table 1. Freeway communication technology and application scenarios

\begin{tabular}{|c|c|c|}
\hline $\begin{array}{c}\text { Communication technology } \\
\text { category }\end{array}$ & Communication technology & Application scenarios \\
\hline $\begin{array}{c}\text { Short-range real-time } \\
\text { communication }\end{array}$ & Bluetooth、RFID、ZigBee、DSRC & $\begin{array}{c}\text { Collision warning, Emergency road conditions, } \\
\text { Emergency call for help, etc }\end{array}$ \\
\hline $\begin{array}{c}\text { Medium-range interactive } \\
\text { communication }\end{array}$ & $\begin{array}{c}\text { WiFi、WiMAX、Mobile cell } \\
\text { technology(LTE、5G, etc.) }\end{array}$ & $\begin{array}{c}\text { Traffic jam, Dynamic speed limit, Temporary } \\
\text { hazard warning, Information entertainment, etc }\end{array}$ \\
\hline
\end{tabular}

Table 2. Comparison of freeway short-range real-time communication technology

\begin{tabular}{|c|c|c|c|c|}
\hline $\begin{array}{c}\text { Communication } \\
\text { technology }\end{array}$ & Bluetooth & RFID & ZigBee & DSRC(WAVE) \\
\hline $\begin{array}{c}\text { Working frequency } \\
\text { band/Hz }\end{array}$ & $2.4 \mathrm{G}$ & $2.45 \mathrm{G} / 5.8 \mathrm{G}$ & $2.4 \mathrm{G}$ & $10 \sim 30(1000)$ \\
\hline $\begin{array}{c}\text { Theoretical } \\
\text { communication } \\
\text { distance/m }\end{array}$ & 15 & 100 & 100 & $6 \sim 27 \mathrm{M}$ \\
\hline $\begin{array}{c}\text { Theoretical } \\
\text { communication rate/bps }\end{array}$ & $1 \mathrm{M}$ & $1 \mathrm{M}$ & $20 \sim 250 \mathrm{k}$ & $<50$ \\
\hline delay/ms & $>100$ & $>200$ & $<500$ & High \\
\hline \begin{tabular}{c} 
Power dissipation \\
\hline
\end{tabular}
\end{tabular}




\begin{tabular}{|c|c|c|c|c|}
\hline Application scenarios & $\begin{array}{c}\text { Voice vehicle } \\
\text { control; Vehicle } \\
\text { condition } \\
\text { monitoring; ETC }\end{array}$ & $\begin{array}{l}\text { Traffic information } \\
\text { collection; } \\
\text { Identification of } \\
\text { vehicles and traffic } \\
\text { signs; Vehicle } \\
\text { localization; ETC }\end{array}$ & $\begin{array}{l}\text { Emergency braking } \\
\text { warning; Forward } \\
\text { collision warning; } \\
\text { Vehicle identification; } \\
\text { Road environment } \\
\text { detection }\end{array}$ & $\begin{array}{l}\text { Forward collision } \\
\text { warning; } \\
\text { Blind spot warning; } \\
\text { Road construction } \\
\text { warning; ETC }\end{array}$ \\
\hline
\end{tabular}

Table 3. Comparison of freeway intermediate-range interactive communication technology $[9,10]$

\begin{tabular}{|c|c|c|c|c|}
\hline $\begin{array}{c}\text { Communication } \\
\text { technology }\end{array}$ & WiFi & WiMAX & LTE & $5 \mathrm{G}$ \\
\hline IEEE standard & $802.11 \mathrm{a}, \mathrm{c}$ & $802.16 \mathrm{e}$ & $3 \mathrm{GPP}$ & $/$ \\
\hline $\begin{array}{c}\text { Theoretical } \\
\text { communication bandwidth }\end{array}$ & $20 \mathrm{MHz}$ & $10 \mathrm{MHz}$ & $20 \mathrm{MHz}$ & $100 \mathrm{MHz}$ \\
\hline $\begin{array}{c}\text { Theoretical } \\
\text { communication rate }\end{array}$ & $6 \sim 54 \mathrm{Mbps}$ & $300 \mathrm{Mbps}$ & $\begin{array}{c}\text { The downward peak } \\
\text { rate is 100Mbps } \\
\text { The upstream peak rate } \\
\text { is 50Mbps }\end{array}$ & $10 \mathrm{Gbps}$ \\
\hline delay & Second level & $<30 \mathrm{~ms}$ & $<50 \mathrm{~ms}$ & $<10 \mathrm{~ms}$ \\
\hline Application scenarios & $\begin{array}{c}\text { Video surveillance; } \\
\text { Traffic information } \\
\text { collection; Service area } \\
\text { information release }\end{array}$ & $\begin{array}{c}\text { Video } \\
\text { surveillance; } \\
\text { Vehicle } \\
\text { positioning }\end{array}$ & $\begin{array}{c}\text { Information } \\
\text { entertainment; Video } \\
\text { surveillance }\end{array}$ & $\begin{array}{c}\text { Danger scene } \\
\text { warning; } \\
\text { Roadside } \\
\text { intellisense }\end{array}$ \\
\hline
\end{tabular}

\section{Existing problems in freeway CVIS communication technology}

\subsection{Application problems of CVIS communi- cation technology}

\subsubsection{Time delay is limited in high speed mobile environment}

The average speed of vehicles on freeways is $60 \% \sim 70 \%$ higher than that of ordinary freeways. High-speed vehicle movement requires faster and more reliable network access and information interaction. Time delay limitation is the main problem in the application of wireless communication technology in freeway communication, It is very important to study the routing protocol based on vehicle location and improve the performance of routing protocol. On the other hand, the channel time variability and Doppler frequency shift caused by high-speed vehicle movement will seriously affect the performance of the mobile communication system, which is particularly prominent for the wireless communication system using OFDM technology in the physical layer. Therefore, how to reduce the influence of Doppler frequency shift on the system is also a hot research issue at present. Literature [11] shows that Doppler Effect and transmission environment changes caused by excessive speed can be dealt with by means of adaptive modulation and power control.

\subsubsection{Packet loss under dense vehicle environment}

Different from the traditional network, the wireless channel quality of MAC layer in vehicular ad-hoc network is seriously affected by road environment and traffic conditions. In freeway environment, MAC protocol in vehicle network is required to support high-speed mobility of vehicles and ensure real-time and reliability of communication. In the case of heavy traffic on freeways such as holidays, channel competition at the MAC layer will be very fierce, resulting in the loss of a large number of packets [12]. How to improve MAC layer protocol to improve the communication quality of V2V and V2I in freeway CVIS is a very important problem.

\subsubsection{Single technology is difficult to meet different application scenarios}

Due to the different communication performance of communication technology, it has its own limitations. For example, the cost and power consumption of RFID and $\mathrm{ZigBee}$ technologies are relatively low. However, due to the limitation of communication rate, the real-time transmission of multimedia information cannot be realized; foreign researches on DSRC technology are relatively mature, but they still cannot overcome the problem of broadcast storm under high traffic density. LTE technology independently researched in China is still in the research stage and has not yet formed a unified standard [13]. There are a variety of communication application modes in the freeway CVIS. Different application scenarios have different requirements on communication rate, delay, reliability and other aspects, meanwhile, cost should also be considered. Therefore, a single wireless access method is difficult to meet the needs of all application scenarios.

\subsubsection{The application practice of CVIS communi- cation technology is insufficient}

The freeway has good road conditions, complete infrastructure, few types of vehicles and simple traffic structure. In developed countries such as the United States, Japan, and Europe, most of the research on CVIS technology begins with freeways. However, China started the research late, and the application of communication technology in freeway V2V and V2I is still in the research stage. At present, in the freeway traffic network, the construction of intelligent roadside facilities is 
insufficient, and whether the vehicle has the communication function is also random. Therefore, the CVIS communication technology has not accumulated enough practical experience, so it is difficult to evaluate the communication effect and its influence on traffic flow effectively.

\subsection{Security issues of CVIS communication network}

\subsubsection{Malicious attacks}

Wireless communication network has the characteristics of openness, mobility, and dynamic changes of topology structure, which makes the network vulnerable to malicious attacks [14], such as information interference, tampering and counterfeiting. Literature [15] shows that there will be information interference phenomenon of the same frequency between communication devices. There are many kinds of communication equipment in the freeway CVIS. When these devices are in the same signal coverage range, the same frequency interference phenomenon will occur. In addition, lawbreakers send high-power same-frequency signals to vehicles and roadside units to interfere with the normal operation of wireless channels [16], which will also lead to the instability of wireless networks. CVIS communication network requires accurate and timely receiving of traffic data. The failure of any relay node will affect the communication of other nodes. Once the attacker sends out the false warning information, it will cause most of the vehicle wireless network to receive the wrong information, which may cause the driver to make a wrong response and lead to the occurrence of traffic accidents.

\subsubsection{User privacy disclosure}

In the CVIS, there will be direct network communication between vehicles and between vehicles and infrastructure. Vehicles can obtain location-based services through shared location, which may lead to the problem of user privacy information disclosure. In order to solve the problem of user privacy leakage in Internet of vehicles, P. Vijayakumar [17] and others proposed efficient anonymous interactive authentication and batch authentication scheme. However, the scheme cannot resist counterfeiting and forgery attacks. Based on this, Qiao [18] proposed a conditional privacy preserving anonymous authentication scheme based on RSU assistance, and pointed out that the solution based on public key infrastructure can effectively protect user privacy.

\subsection{Development trend of freeway CVIS communication technology application}

4.1.1 Emerging wireless communication
technologies

With the rapid development of 5G technology, the Internet of Vehicles has ushered in a new historical opportunity. Literature [19] proposes a 5G car networking architecture that can be connected and integrated with multiple networks based on the current challenges of vehicle networking. In December 2018, the Beijing Bureau of Economy and Information Technology issued and implemented the action plan for $5 \mathrm{G}$ car networking. In July 2019, at the summit forum "Creating a New Life of Travel" held in Liuzhou, Guangxi, SAIC-GM-Wuling announced that the first intelligent net-connected vehicle with $5 \mathrm{G}$ communication, V2X, driverless vehicle and remote driving control in China was officially launched for public test. At present, the test road has completed the layout of $95 \mathrm{G}$ base stations [20]. Instead of deploying roadside infrastructure alone, $5 \mathrm{G}$ Internet of Vehicle can connect with small cellular networks, RSU and other infrastructure units to build a multi-network access and fusion architecture. In the future, 5G technology will become an important condition for the realization of Internet of Vehicles, leading a new era of Expressway Internet of Vehicles.

\subsubsection{Coexistence of multiple heterogeneous communication networks}

Heterogeneous wireless network convergence is an important trend in the development of freeway CVIS communication technology. In order to adapt to the requirements of different communication application modes and scenarios on communication rate, delay, reliability and so on, freeway wireless access network presents the situation of coexistence of multiple technologies. In 2016, Qualcomm released a reference platform for intelligent connected vehicles, which provides users with data migration mode from DSRC to hybrid/cellular V2X and 4G LTE to 5G. It supports both long-term Evolution (LTE) and IEEE 802.11p for DSRC. The future freeway communication network will be a heterogeneous wireless network with multiple access networks. Based on the heterogeneous network fusion, we can choose the appropriate communication mode and protocol according to the different application scenarios of freeway communication, and each network can integrate and cooperate with each other to achieve the desired effect.

\subsubsection{Strengthen the construction of freeway informatization}

At present, the development trend of Internet of Vehicles in China is good, but it is still in the primary stage. In 2017, Chongqing builds intelligent vehicle reliability test area at $5.9 \mathrm{GHz}$ LTE-V2X test point, which can be used for freeway Vehicle-Infrastructure cooperation, safe driving and other tests [21]. In 2018, Zhejiang Province plans to build Hangzhou Shaoxing Ningbo Expressway, the first super Expressway in China, and strive to complete and test run it in 2022. This expressway will build an international Internet of Vehicles system to fully support automatic driving [22]. In addition, the Hangzhou Bay Smart freeway network is expected to take shape in 
2023. The development of future communication technologies will help drive the construction of a new group of communication base stations and smart road side units, improve the smart road side system of the freeway, and accelerate the construction process of smart freeway [23].

\subsection{Development trend of freeway CVIS communication network security}

Wireless interference may cause sudden errors in transmission information and cause error codes. Research shows that channel coding combined with interleaving technology is the mainstream scheme to suppress wireless interference [24]. In the future, CVIS communi- cation network will combine the interleave technology and adopt channel coding technologies such as $\mathrm{BCH}$ code, Turbo code and RS code to effectively prevent interference from interference sources to wireless network communication.

The attacker tampers and counterfeits the information transmitted by vehicles and roadside units, which may affect the judgment of drivers and lead to traffic accidents. In the future, the freeway CVIS communi- cation technology will adopt the signal modulation method with good concealing property to prevent attackers from intercepting the information, and encrypt the information transmitted by vehicles and roadside units to prevent attackers from interfering with data packets used by information transmission nodes. In addition, due to the high-speed movement of vehicles, the communication network topology and road environ- ment are constantly changing. The communication network will face a variety of unknown attacks, network intrusion detection is particularly important [25]. In the future, such methods as machine learning and deep learning can be used to automatically monitor network intrusion and deal with unknown attacks, so as to ensure the security of freeway CVIS communication network.

\section{References}

1. Z.G. Xu. The main research content of intelligent roadside system [EB/OL]. http://www.docin.com/p-17044 8569.html.(2019).

2. K. Zheng, Q. Zheng, P. Chatzimisios, et al. Heterogeneous vehicular networking: A survey on architecture, challenges and solutions. IEEE Communications Surveys \& Tutorials, 17(4):1, (2015).

3. Y. Han. The discussion of connected vehicles application based on new bluetooth technology standard. The 8th China intelligent transportation annual conference. 139-143, (2013).

4. M. Karatsoli, M. Margreiter, M. Spangler. Bluetooth-based travel times for automatic incident detection: A systematic description of the characteristics for trafficmanagement purposes. Transportation Research Procedia, 24(3):204-211, (2017).

5. J. Tian. Research on detecting device of traffic perception system based on Bluetooth. Communications Science and Technology Heilongjiang, 41(05): 216-217, (2018).

6. J.J. Chen, B.Y. Shan. Survey on $5.8 \mathrm{GHz}$ electronic toll collection technologies. Journal of Tongji University
(Natural Science), 38(11):1675-1681, (2010).

7. C.M. Chou, C.Y. Li, W.M. Chien, et al. A feasibility study on Vehicle-to-Infrastructure communication: WiFi vs. WiMAX. Tenth International Conference on Mobile Data Management: Systems, 397-398, (2009).

8. J.J. Wu. The application of $5 G$ technology in intelligent transportation. Electronic Technology and Software Engineering, (16):34-35, (2019).

9. M. Haider, A.H. Turki, J.A. Muthanna, LTE capacitye stimation with changing different planning parameter.J ournal of Communications,15(09):687-692, (2020).

10. A. Farhan, Y.G. He, G.L. Shi, et al. Future generation spectrum standardization for $5 G$ and Internet of Things. Journal of Communications, 15(03): 276-282, (2020).

11. X.L. Feng, L. Xu, N.N. Quan, et al. An Analysis of ZigBee Vehicle-Road Communication performance in the Internet of Vehicles. Journal of Transport Information and Safety. 33(03):71-76, (2015).

12. A.T. Akabane, L.A. Villas, E.R.M. Madeira. Data dissemination in Freeway scenarios using car-to-car communication. 2013 International Conference on Connected Vehicles and Expo (ICCVE). IEEE, (2013).

13. F. Fang. Research of cooperative Vehicle-infrastructure communication technology. China Intelligent Transportation Systems Association, 1028-1033, (2016).

14. Y.M. Harun, H.M. Furqan, et al. Cognitive security of wireless communication systems in the physical layer. Wireless Communications and Mobile Computing,(2017)

15. K. Yamazaki, Y. Sugiyama, Y. Kawahara, et al. Interference cancellation for coexisting wireless data and power transmission in the same frequency. Journal of Physics: Conference Series, 2014, 557:012004.

16. X.R. Han, Z.M. Wang. Specific analysis of wireless communication network security. Scientific and Technological Innovation, 2019,(19):79-80.

17. P. Vijayakumara, V. Changb, L.J. Deboraha, et al. Computationally efficient privacy preserving anonymous mutual and batch authentication schemes for vehicular ad hoc networks. Future Generation Computer Systems, 78(2): 943-955, (2016).

18. R. Qiao. Research on anonymous authentication technology for VANETs. Xi'an: Chang'an University, (2019).

19. L.M. Wang, X.L. Liu, C.X. Li, et al. Overview of internet of vehicles for $5 G$. Chinese Journal of Network and Information Security, 2(06):1-12, (2016).

20. Souhu. $5 G$ wave is coming! New baojun opened the country's first public test road $5 G$ base station group. http://www.sohu.com/a/330265437_100119460.(2019)

21. Souhu. Zhejiang will build the first superhighway in China to support automatic driving and mobile wireless charging. http://www.sohu.com/a/224122358_69135 1.(2019)

22. X.J. Liu, L.L. Dai, L. Gao. Progress and prospect of vehicle networking pilot project. China Radio, 08:30-32, (2018).

23. J.S. Zhang, B. Li, X.J Wang, et al. Design of architecture and development roadmap of Smart freeway. Journal of freeway and Transportation Research and Development, 35(01):88-94, (2018).

24. C.K. Wang. Study of turbo codes for Anti-burst interference based on matlab. Zhengzhou: Zhengzhou University,(2013)

25. X.H. Li, C. Zhong, Y. Chen, et al. Survey of internet of vehicles security. Journal of Cyber Security, 4(03):17-33, (2019). 